\title{
At last, direct evidence that lipoxygenases play a role in atherogenesis
}

\author{
Daniel Steinberg \\ Department of Medicine 0682, University of California-San Diego, 9500 Gilman Drive, La Jolla, \\ California 92093, USA. Phone: (619) 534-0569; Fax (619) 534-2005; E-mail: dsteinberg@ucsd.edu.
}

The possibility that oxidative modification of LDL might be a key step in the genesis of the atherosclerotic lesion was suggested more than 15 years ago, and a great deal of evidence has accumulated to support this hypothesis $(1,2)$. The pivotal evidence comes from studies in animal models of atherosclerosis, showing that the administration of appropriate antioxidants at appropriate levels of intake significantly slows the progression of the disease (summarized in ref. 3). This protection is associated with a marked decrease in the susceptibility of circulating LDL to oxidation ex vivo and with decreases in markers of lipid oxidation such as isoprostanes, lipid hydroperoxides, and their breakdown products. Several different animal models have been studied (LDL receptor-deficient rabbits and mice; cholesterol-fed rabbits, hamsters and monkeys; apo E-deficient mice), and several different antioxidants have been shown to be effective (probucol; a metabolite of probucol; butylated hydroxytoluene; diphenylphenylenediamine; and vita$\min \mathrm{E})$. The magnitude of the effects in these animal models is striking - the rate of progression of lesions has been slowed by $50 \%-80 \%$. Consequently, there is intense interest in the question of whether antioxidants will work in the human disease. If they do, such treatment might be additive to, or possibly even synergistic with, treatment with cholesterol-lowering agents.

Despite an enormous amount of work on the mechanisms of LDL oxidation, we still do not know which enzyme systems or which nonenzymatic oxidative mechanisms are responsible for oxidation of LDL in vivo, nor which radical species are involved. In principle, any enzyme system that generates free radicals could contribute to LDL oxidation. Studies in vitro have identified a long list of candidates, including NADPH oxidase, myeloperoxidase, P450, the mitochondrial electron transport system, xanthine oxidase, and lipoxygenase(LO), which was first implicated in
1988 by the demonstration that soybean (LO) could oxidize LDL in vitro to a form recognized by macrophage scavenger receptors (4). However, there is little or no evidence on the relative importance of these candidate systems in vivo.

In this issue of the JCI, Cyrus et al. (5) report results of a critical transgenic experiment that strongly supports a role for 12/15-LO in atherogenesis. They show that homozygous disruption of the LO gene diminishes atherosclerosis in apo E-deficient mice. At 15 weeks, the aortic lesion area in control animals (apo E knockouts, but wildtype with respect to LO) was 15,700 $\mathrm{mm}^{2}$, whereas in the double knockout mice it was only $198 \mathrm{~mm}^{2}$. In other words, in the absence of LO there was virtually no initiation of lesions during the first 15 weeks. At the end of 1 year, however, lesions had progressed in the LO knockout animals, and the difference was much less striking (approximately a $50 \%$ decrease in lesion area) but still highly significant $(P<0.0001)$. These studies provide the first direct and specific evidence for a role of $\mathrm{LO}$ in atherogenesis. Sendobry et al. (6) have previously reported an antiatherosclerotic effect of an inhibitor of $15-\mathrm{LO}$ in cholesterol-fed rabbits. However, it was not explicitly established to what extent 15-LO activity was in fact inhibited in vivo, or that the inhibitor drug was free of other biologic effects.

These new knockout results also provide important general support for the validity of the oxidative modification hypothesis and its relevance in mouse models. Four studies have now shown that antioxidants slow the development of lesions in the mouse: one using diphenylphenylenediamine in the apoE knockout model (7), one using a synthetic analogue of probucol in LDL-R knockout mice (8), one using vitamin $\mathrm{E}$ in the apo E knockout model (9), and one using a metabolite of probucol in apo E, LDL-R double-knockout animals (10). Considering these results together with those of Cyrus et al., it can now be safely concluded that oxidation of LDL is a significant factor in murine models of atherosclerosis, as it is in other animal models, and that the worsening of atherosclerosis observed in probucoltreated mice (11) probably reflects some other biologic property of probucol peculiar to the mouse, rather than a basic difference in pathogenesis.

In the studies by Cyrus et al., it should be noted that the apo E-deficient mice that were heterozygous with regard to LO did not show any amelioration of atherosclerosis. Interestingly, while the peritoneal macrophages from the mice homozygous for the LO knockout generated almost no LO enzymatic products from arachidonic acid, the macrophages from heterozygotes oxidized arachidonic acid almost as well as did wild-type macrophages. The implication is that the level of LO activity had to be reduced by more than $50 \%$ before one could observe either a decrease in LO-mediated arachidonate oxidation or any effect on the progress of atherosclerosis. Of course, we cannot be sure that the reduction in rate of generation of oxidized products is $50 \%$. There could be compensatory changes in the functioning of the normal LO allele to enhance its expression, or there could be rate-limiting factors that determine the actual rate of product formation. Putting these possibilities aside for the moment, however, these new findings may be relevant to an apparent anomaly observed some years ago with respect to the role of LO in oxidation of LDL by cells in culture. Parthasarathy et al. (12) showed that an analogue of arachidonic acid (ETY) inhibited LO and reduced the rate of LDL oxidation by cultured endothelial cells. However, very high concentrations were needed, and later studies by Sparrow and Olszewski (13) showed that the concentrations used would have been sufficient to inhibit LO by $90 \%$ or more. It is possible that the levels of $\mathrm{LO}$ in some cells do not become rate limiting for oxidation of LDL until those levels are reduced by more than $50 \%$. This is 
not the case in cells that don't express LO and therefore start with very low rates of LDL oxidation. For example, it has been shown that expressing LO in fibroblasts increases their ability to oxidize LDL in the medium $(14,15)$, possibly by transferring lipid hydroperoxides generated in the cell membrane to the LDL in the medium.

It should not necessarily be concluded that the deletion of LO in Cyrus' knockout mice decreased lesion progression just by sparing LDL from oxidation (5). This enzyme generates a number of biologically potent products that can modulate redox-sensitive signaling pathways (16). Thus, additional mechanisms may participate. However, Cyrus et al. also report that the titers of autoantibodies against oxidized LDL and against malondialdehyde-LDL were significantly lower in the homozygous LO knockout animals. While indirect, these findings help support the interpretation that the rate of oxidation of LDL in vivo was indeed reduced, particularly since the plasma LDL concentrations were not different.

Still to be explained are the apparently conflicting results of Shen et al. (17), who found that selective overexpression of LO in monocyte/macrophages actually reduced lesion area by almost $50 \%$ in cholesterol-fed rabbits. Their failure to see an increase in the severity of lesions might be explicable on the basis discussed above - namely, that even though there is an increased expression of enzyme protein, there may not be a corresponding increase in the actual rate of generation of LO products. Also, the overexpression in this case was limited to macrophages, and their role in the generation of oxidized LDL in vivo may not be dominant. Neither of these explanations is particularly attractive; and in any case, there remains the problem of explaining why the animals actually showed reduced lesion development. Kuhn and Chan (16) have speculated that LO may exert protective effects relating to the expression of redox sensitive genes and/or that the effects of LO at different stages of lesion development may differ considerably.

In summary, the results of Cyrus et al., taken together with the previous work indicating the involvement of LO in oxidation of LDL, would appear to move it from the candidate list to the "real" list. The quite remarkable inhibition of early lesion formation suggests that $\mathrm{LO}$ is a major player in atherogenesis. On the other hand, the lesions do progress in the LO-deficient animals, so additional factors are undoubtedly involved. Would the addition of antioxidants to the diet of these LO knockout animals further inhibit lesion progression? If so, it would imply that alternative systems for LDL oxidation are involved. It should be noted that the cholesterol levels in the animals studied were over $500 \mathrm{mg} / \mathrm{dL}$. Would the protective effect of knocking out LO be even more dramatic in animals with more modest elevations of blood cholesterol?

1. Steinberg, D., Parthasarathy, S., Carew, T.E., Khoo, J.C., and Witztum, J.L. 1989. Beyond cholesterol. Modifications of low-density lipoprotein that increase its atherogenicity. N. Engl. J. Med. 320:915-924.

2. Steinberg, D., and Witztum, J.L. 1990. Lipoproteins and atherogenesis. Current concepts. JAMA. 264:3047-3052.

3. Steinberg, D. 1997. Oxidative modification of LDL and atherogenesis. The 1995 Lewis A. Conner Memorial Lecture. Circulation. 95:1062-1071.
4. Sparrow, C.P., Parthasarathy, S., and Steinberg, D 1988. Enzymatic modification of low density lipoprotein by purified lipoxygenase plus phospholipase A2 mimics cell-mediated oxidative modification. J. Lipid Res. 29:745-753.

5. Cyrus, T, et al. 1999. Disruption of the $12 / 15$ lipoxygenase gene diminishes atherosclerosis in apolipoprotein E-deficient mice. J. Clin. Invest. 103:1597-1604.

6. Sendobry, S., et al. 1997. Attenuation of dietinduced atherosclerosis in rabbits with a highly selective 15-lipoxygenase inhibitor lacking significant antioxidant properties. Br. J. Pharmacol. 120:1199-1206

7. Tangirala, R.K., et al. 1995. Effect of the antioxidant N,N'-diphenyl 1,4-phenylenediamine (DPPD) on atherosclerosis in apoE-deficient mice. Aterioscler. Thromb. Vasc. Biol. 15:1625-1630.

8. Cynshi, O., et al. 1998. Antiatherogenic effects of the antioxidant BO-653 in three different animal models. Proc. Natl. Acad. Sci. USA. 95:10123-10128.

9. Pratico, D.T., Tangirala, R.K., Rader D.J., Rokach, J., and FitzGerald, G.A. 1999. Vitamin E suppresses isoprostane generation in vivo and reduces atherosclerosis in apoE-deficient mice. Nat. Med. 10:1189-1192.

10. Witting, P.K., et al. 1999. Inhibition by a coantioxidant of aortic lipoprotein lipid peroxidation and atherosclerosis in apolipoprotein $\mathrm{E}$ and low density lipoprotein receptor gene double knockout mice. FASEB J. 13:667-675.

11. Zhang, S.H., et al. 1997. Paradoxical enhancement of atherosclerosis by probucol treatment in apolipoprotein E-deficient mice. J. Clin. Invest. 99:2858-2866.

12. Parthasarathy, S., Wieland, E., and Steinberg, D. 1989. A role for endothelial cell lipoxygenase in the oxidative modification of low density lipoprotein. Proc. Natl. Acad. Sci. USA. 86:1046-1050.

13. Sparrow, C.P., and Olszewski, J. 1992. Cellular oxidative modification of low density lipoprotein does not require lipoxygenases. Proc. Natl. Acad. Sci. USA. 89:128-131.

14. Benz, D., et al. 1995. Enhanced levels of lipoperoxides in low density lipoprotein incubated with murine fibroblasts expressing high levels of human 15-lipoxygenase. J. Biol. Chem. 270:5191-5197.

15. Ezaki, M., Witztum, J.L., and Steinberg, D. 1995. Lipoperoxides in LDL incubated with fibrob lasts that overexpress 15-lipoxygenase. J. Lipid Res. 36:1996-2004.

16. Kuhn, H. and Chan, L. 1997. The role of 15-lipoxygenase in atherogenesis: pro-and antiatherogenic actions. Curr. Opin. Lipidol. 8:111-117.

17. Shen, J., et al. 1996. Macrophage-mediated 15lipoxygenase expression protects against atherosclerosis development. J. Clin. Invest. 98:2201-2208. 